\title{
Programme D'entrainement Pour Développer Les Compétences De La Communication Écrite Chez Les Étudiants Du Cycle Secondaire
}

\author{
Mohamed Salah Abdelaal
}

\begin{abstract}
La recherche actuelle a pour but de développer quelques compétences de la communication écrite (compréhension et la production) chez les étudiants de la deuxième année du cycle secondaire à travers l'utilistion d'un programme d'entrainement en examinant son effet. L'échantillon de cette recherche se compose de 30 étudiants parmi ceux de la deuxième année secondaire constituant le groupe expérimental de cette recherche. L'outil et le matériel de la présente recherche portent sur un test des compétences de la communication écrite (compréhension et production) et un programme d'entrainement.

Après avoir enseigné le programme d'entrainement proposé, les résultats ont conduit à la vérification de l'hypothèse de la recherche:

"Il y a une différence significative statistiquement au niveau $P \leq 0,05$ entre les moyennes des notes d'étudiants de l'échantillon de la recherche au prélposttest des compétences de la communication écrite en faveur du post test».
\end{abstract}

\section{Introduction}

L'apprentissage d'une langue, comme tout apprentissage, est un processus, personnel, social, et se manifeste à travers la responsabilisation de l'apprenant face à la construction de ses savoirs et à leur utilisation dans des contextes de plus en plus variés et complexes.

L'enseignement/apprentissage du français langue étrangère vise à développer chez les apprenants les compétences communicatives à l'oral (écouter/parler) et à l'écrit (lire/écrire). Ces apprenants seront amenés, progressivement, à communiquer à l'oral comme à adaptées à leur niveau cognitif. (Alrabadi, 2011: 15-34).

Dans cette optique, la compréhension écrite ouvre à tous les apprenants la possibilité de se documenter et d'avoir accès à communiquer en aidant les apprenants à penser, à réfléchir et à comprendre. On lit, donc, pour savoir ce que font les autres, ou ce qu'ils deviennent; pour savoir d'un jour à l'autre ce qui se passe chez nous, dans notre pays et ce qui se passe d'ailleurs, dans les autres pays du monde (Barychnikov, 1995) cité par Gabr (2007: 11). 
En fait, la compréhension écrite est considérée comme une interaction entre les signes, le sens et le contexte. Ainsi, elle contient le choix du sens adéquat, l'organisation des idées lues et les réutiliser dans d'autres situations de communication.

Donc, la compréhension écrite se considère une opération complexe ayant des processus mentaux et cognitifs. Elle est également une activité complexe de traitement d'informations graphiques dont l'objectif est d'extraire la signification d'une phrase écrite. La compréhension écrite est donc le produit de deux ensembles d'opérations: d'une part, des activités spécifiques, le décodage, destinées à analyser puis à saisir l'information graphique pour accéder à l'information linguistique. D'autre part, elle constitue une série d'activités mentales de traitement des significations qui relèvent de la compréhension proprement dit. Les deux composantes essentielles de la lecture (décodage et compréhension) fonctionnent en interaction.

Quant à la production écrite, elle représente un acte qui amène l'apprenant à exprimer ses idées, ses sentiments, ses interêts pour se communiquer. Ecrire, c'est donc produire une communication à travers d'un texte. La production écrite n'est pas une aptitude isolée, c'est une activité subsidiaire à la lecture parce que l'acquisition de son processus est liée à la lecture par laquelle les apprenants manipulent les concepts reliés en langage écrit. En bref, lire pour écrire. Enfin, ces deux compétences, la lecture et l'écriture (ici la commuication écrite), se développent parallèlement.

Ainsi, écrire un texte, c'est produire du sens afin de réaliser un but communicationnel. Dans ce sens, ce qui prime le plus est le sens du texte plutôt que la forme, la transmission du message plutôt que la correction absolue. Par conséquent, elle est considérée comme l'une des compétences que les étudiants doivent maîtriser pour pouvoir se communiquer (Ali, 2014: 91-92).

On

Selon Mohamed (2005: 103106), la communication écrite se compose des deux axes: la compréhension écrite et la production écrite:

\section{- La compréhension écrite}

Par la compréhension écrite, l'apprenant obtient l'information, apprend des modèles de communication, correspond avec les autres, il peut agir et intervenir dans la vie de la communauté.

\section{- La production écrite}


Elle est un processus de production écrite et considérée comme une opération mentale impliquant trois étapes (la planification, la mise en texte et la révision), et comme une activité mentale supposant la mise en œuvre d'un ensemble de connaissances langagières et de différents processus mentaux.

La communication écrite touche pour l'aspect linguistique, en premier lieu à la syntaxe de la langue de départ. Par ailleurs, elle s'enrichit le vocabulaire chez l'apprenant.

Dans cette optique, l'acte de lecture et d'écriture est considéré comme un processus de construction de sens et de communication. De plus, la compétence de la communication écrite (compréhension et production écrites) est une acrivité à part entière et se traduit par la capacité à pratiquer la langue dans diverses activités: raconter, décrire, expliquer, ... etc (Esmat, 2016: 21).

Dans ce sens, savoir communiquer, c'est établir un lien entre l'écriture avec la lecture. C'est la communication écrite qui est en effet, mettre en place un nouveau langage qui s'adresse à un lecteur demande une expression plus concise et pertinente. Elle crée un lien incontestable entre son auteur et le lecteur.
Dans cette perspective, on constate que le développement des compétences de la communication écrite (compréhension et production écrites) chez les étudiants du cycle secondaire, a constitué l'objectif de plusieurs études effectuées dans le domaine de la didactique du FLE en Egypte comme: Abdel Aal (2016), Abd El Salam (2007), Al Banoui (2018), Ali (2014), Awad (2013), Badary (2017), Chalabi (2014), El-Ahwal (1991), El-Chahat (2000), El safy (2005), Esmat (2016), ), Fouad (2014), Mohamed (2005), Mahfouz (2009), Nassar (2017) et Sobhi (2010) .

Le chercheur consulte ces études antérieures pour confirmer l'existence du problème de la recherche $\mathrm{du}$ fait qu'elles nous aident à déterminer les compétences nécessaires à l'amélioration la communication écrite (la compréhension et la production) chez les étudiants du cycle secondaire comme:

- identifier le sens des mots à travers le contexte;

- décrire une illustration du texte en utilisant des propres mots;

- cerner l'intention de communication écrite;

- résumer un texte écrit;

- construire des phrases correctes grammaticalement et orthographiquement; 
- donner et demander des informations;

- exprimer des attitudes (accord/désaccord, permission, préférences, goût, excuses);

- établir des relations sociales (s'adresser aux gens, saluer, présenter, prendre congé);

Ces études nous présentent également de différentes stratégies de remédier les compétences de la communication écrite, elles nous indiquent les certaines (unités, programmes, modèles...etc) et nous fournissent les outils de l'évaluation des compétences de la communication écrite.

Malgré l'importance vitale du développement des compétences de la communication écrite (compréhension et production écrites, il y a encore une faiblesse dans ces compétences chez les étudiants du cycle secondaire.

Ainsi, pour développer les compétences de la communication écrite, et par conséquent surmonter cette faiblesse, les études antérieures ont recommandé la nécessité de l'utilisation de nouvelles méthodes visant à développer ces compétences et transmettant le plaisir d'apprendre grâce à une démarche riche en activités variées. De ce fait, la didactique des langues se propose d'utiliser des programmes d'entrainement.

\section{Sensibilisation au problème}

La sensibilisation au problème de la recherche actuelle consiste à /aux:

- Les études antérieures consultées.

- L'expérience personnelle du chercheur.

- L'étude pilote.

\section{-Les études antérieures consultées}

Le chercheur a consulté plusieurs études touchant le domaine de la communication écrite (compréhension écrite et production écrite) comme: Abdel Aal (2016), Abd El Salam (2007), Al Banoui (2018), Ali (2014), Awad (2013), Badary (2017), Chalabi (2014), El-Ahwal (1991), El-Chahat (2000), El safy (2005), Esmat (2016), Fouad (2014), Mohamed (2005), Mahfouz (2009), Nassar (2017) et Sobhi (2010). Toutes ces études ont montré que le niveau des apprenants est faible en ce qui concerne les compétences de la communication écrite (compréhension et production écrites) par rapport au domaine des langues étrangères.

\section{- L'expérience personnelle}

Ces résultats se sont mises en d'accord avec l'observation et l'expérience personnelles du chercheur, en tant que premier enseignant de français au secteur 
gouvernemental, sur l'existence d'une faiblesse chez les étudiants de la 2ème année secondaire en ce qui concerne les compétences de la communication écrite.

\section{- L'étude pilote}

En vue de valider les observations remarquées, le chercheur a effectué une étude pilote visant a diagnostiquer le niveau actuel d'un échantillon du public ciblé. Tout d'abord, le chercheur a élaboré un test concernant certaines compétences de la communication écrite (compréhension écrite et production écrite) et l'a appliqué ensuite sur un échantillon composé de 15 étudiants des étudiants de la 2ème année secondaire. Les résultats de cette étude pilote ont démontré que le niveau des étudiants ciblés est faible en ce qui concerne les compétences de la communication écrite mésurées (compréhension écrite et production écrite). Ces résultats sont présentés dans le tableau suivant:

Tableau (1) Résultats du test des compétences de la communication écrite de l'étude pilote

\begin{tabular}{|l|c|c|}
\hline \multicolumn{1}{|c|}{ Compétences de la communication écrite } & $\begin{array}{c}\text { Nombre } \\
\text { des } \\
\text { étudiants }\end{array}$ & $\begin{array}{c}\text { \% des } \\
\text { réponses } \\
\text { correctes }\end{array}$ \\
\hline 1- Se rappeler des mots emmagasinés en mémoire & 15 & $40 \%$ \\
\hline 2- Illustrer une image du texte écrit. & 15 & $45 \%$ \\
\hline 3- Identifier le sens d'une phrase du texte écrit & 15 & $47 \%$ \\
\hline 4- Résumer un texte écrit & 15 & $33 \%$ \\
\hline $\begin{array}{l}\text { 5- Utiliser le lexique donné et adapté à une situation de } \\
\text { communication }\end{array}$ & 15 & $27 \%$ \\
\hline $\begin{array}{l}\text { 6- Décrire une situation par écrit (par ex. décrire une image, } \\
\text { des objets et des personnes) }\end{array}$ & 15 & $33 \%$ \\
\hline 7- Annoncer ce qu'on va faire/ce qu'on ne va pas faire & 15 & $26 \%$ \\
\hline $\begin{array}{l}\text { 8- Communiquer par écrit une variété de messages (par ex. } \\
\text { remerciements, d'accord/désaccord, vœux de santé, } \\
\text { demander la permission et présenter et se présenter); }\end{array}$ & 15 & $27 \%$ \\
\hline
\end{tabular}

Les résultats indiqués dans le tableau no (1) mettent en relief l'existence d'une faiblesse chez les étudiants de la $2^{\text {ème }}$ année secondaire, membres de l'échantillon par rapport aux compétences de la communication écrite en FLE quelle soit au niveau de la compréhension écrite qu'au niveau de la production écrite. 
3. Problématique de la recherche

Le chercheur pourrait formuler la problématique de la recherche actuelle dans la question principale suivante: Quel est l'impact d'un programme d'entrainement pour développer certaines compétences de la communication écrite (compréhension et production) chez les étudiants de la deuxième année du cycle secondaire?

Pour pouvoir répondre à cette question principale, il est nécessaire de répondre aux sousquestions suivantes:

- Quelles sont les compétences de la communication écrite (compréhension et production) nécessaires aux étudiants de la deuxième année du cycle secondaire?

- A quel niveau les étudiants de la deuxième année secondaire possèdent-ils les compétences de la communication écrite (compréhension et production)?

- Quel est le programme d'entrainement pour développer les compétences de la communication écrite (compréhension et production) chez les étudiants de la deuxième année du cycle secondaire?

- Quel est l'mpact du programme d'entrainement pour développer certaines compétences de la communication

écrite

(compréhension et production) chez les étudiants de la deuxième année du cycle secondaire?

\section{Hypothèse de la recherche}

La recherche actuelle essaye de vérifier l'hypothèse suivante:

- «Il y a une différence significative statistiquement au niveau $\mathrm{P} \leq 0,05$ entre les moyennes des notes d'étudiants de l'échantillon de la recherche au pré/post test des compétences de la communication écrite (compréhension et production) en faveur du post test».

\section{Objectifs de la recherche}

Cette recherche vise à réaliser les objectifs suivants

- Développer certaines compétences de la communication écrite (compréhension et production) chez les étudiants de la deuxième année secondaire.

- Mesurer l'impact d'un programme d'entrainement dans le but de développer ces compétences.

\section{Délimites de la recherche}

La recherche actuelle se limite à :

- Un échantillon des étudiants de la deuxième année secondaire dans une école secondaire au district de Timi Al Amdid au gouvernorat de Dakhlia. 


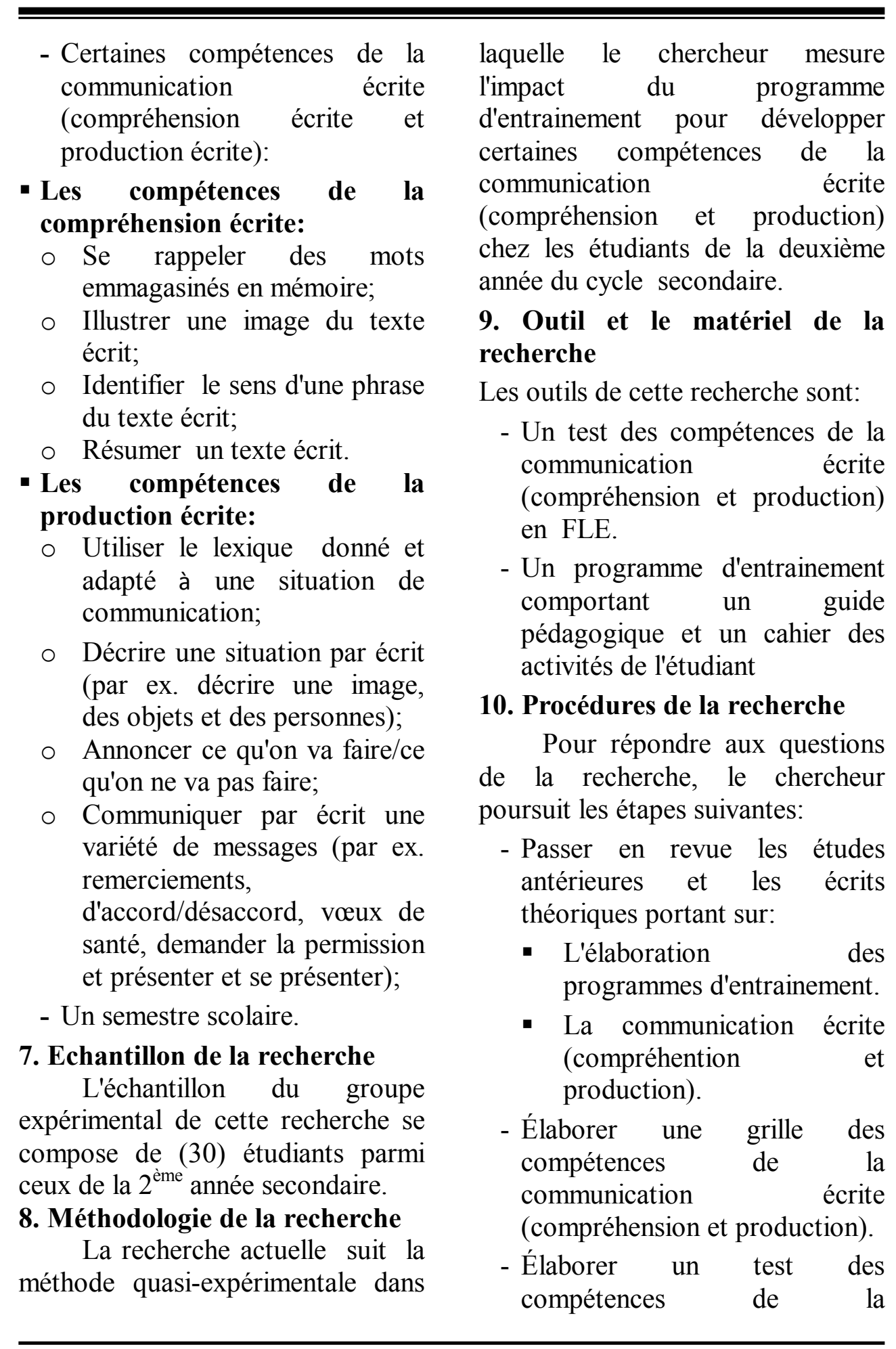


communication écrite pour vérifier le niveau des étudiants de la deuxième année du cycle secondaire par rapport aux compétences de la communication écrite (compréhension et production).

- Élaborer un programme d'entrainement

- Présenter les outils et le programme d'entrainement à un jury de spécialistes du domaine afin de déterminer la validité apparente, les modifier à la lumière de son avis et les mettre dans leur forme finale.

- Standardiser le test (calculer la validité et la fidélité).

- Choisir l'échantillon de la recherche.

- Pré-application du test des compétences de la communication écrite (compréhension et production) sur le groupe expérimental de la recherche.

- Enseigner le programme d'entrainement sur le groupe expérimental de la recherche.

- Post-application du test des compétences de la communication écrite (compréhension et production) sur le groupe expérimental de la recherche.

- Analyser et interpréter les résultats de la recherche.
- Présenter les recommandations et les suggestions de la recherche.

\section{Terminologies de la recherche}

On va exposer les terminologies de cette recherche comme suit:

\section{Le programme d'entrainemnt}

C'est un ensemble qui implique un groupe d'objectifs, d'unités, de stratégies d'enseignement et d'outils d'évaluations dans le but de développer les compétences de la communication écrite (compréhension et production) assignées.

À partir de la définition cidessus, on peut définir notre programme d'entrainemnt comme la capacité des apprenants à comprendre un texte et à utiliser l'écriture pour utiliser les fonctions de communications précédentes audessus dans la vie quotidienne. On étudie la capacité des étudiants concernant la compétence de communication (compréhension écrite: comprendre des textes simples, une invitation simple, se faire une idée du contenu d'un texte informatif, descriptif,...etc et production écrite: produire des mots simples, copier des mots donnés, écrire un message électronique simple, un dialogue et réviser et améliorer l'écrit qu'on a produit. 


\section{La compétence de la communication écrite}

D'après Mohamed (2005: 12), la compétence de la communication écrite est définie comme la capacité mentale qui signifie la facilité d'achever un travail écrit en respectant les conditions de situation. Elle un moyen qui suppose aux individus un dominateur commun de la compréhension et de la production écrite sur des sujets communs à traiter entre eux ou bien avec d'autres sociétés afin de trnasmettre une connaissance ou une culture.

La compréhension écrite est, en fait, en fonction des connaissances du lecteur, de sa capacité à traiter les marques linguistiques (lexicales, syntaxiques et textuelles), mises en oeuvre par le scripteur, mais aussi dans les buts qu'il poursuit à travers sa lecture. C'est l'étape du codage où le lecteur accède à la signification du mot en recherchant dans le lexique mental, sorte de dictionnaire interne où sont rangées les informations lexicales (orthographe, signification, etc...). L'analyse sémantique consiste à construire une signification globale la phrase puis le sens global du texte entier (Mahfouz, 2009: 67).

En se référant à la théorie de communication, Deschênes (1988: 19) définit l'écriture comme suit: « ecrire un texte c'est tracer des lettres, de mots, des phrases, mais aussi et surtout c'est élaborer un message qui veut transmettre la pensée de l'auteur donc nécessairement tout un travail cognitif d'élaboration, de structuration de l'information qui est le résultat d'interlocuteur et le scripteur».

Selon Chartrand (2008), cité par (Ali, 2014: 91), écrire un text "c'est une activité langagière et pratique sociale qui consiste à produire du sens original à l'aide d'outils sémiotique (genres de textes et langue), graphiques et matériel. Cela implique de s'approprier et de mettre en œuvre de façon circonstanciée divers systèmes (genre textuel; grammaire du texte, grammaire de la phrase: syntaxe, orthographe; lexique).

Et pour cette recherche, la communication écrite est le processus où l'apprenant associe entre la réalité du texte donné et les expériences antérieures qu'il a dans sa mémoire, il organise entre les deux pour comprendre ce contexte en ajoutant de nouvelles expériences autonomes et c'est également le processus où l'apprenant utilise la langue en écrivant en situations de communication par des phrases simples grammaticalement lexicalement et correctes afin de 
réaliser la communication par et avec l'écrit.

\section{Cadre théorique}

Le cadre théorique de cette recherche comporte deux volets principaux: la compréhension écrite et la production écrite.

\subsection{La compréhension écrite}

On va exposer l'importance de de la compréhension écrite, ses étapes et son lien avec les autres compétences langagières et ses compétences.

\subsubsection{L'Importance de la compréhension écrite}

L'acte de lire est perçu comme un processus plus cognitif que visuel, comme un processus actif et interactif, comme un processus de construction de sens et de communication (Giasson, $2007: 6$ ).

Quelques linguistes confirment que la compréhension écrite est considérée comme un instrument le plus effectif afin d'acquérir les matières à enseigner et qu'il y a une grande corrélation entre la compréhension écrite et l'acquisition, pour conclure, l'apprenant qui peut lire, peut aussi acquérir et progresser dans son enseignement. Elle joue également un rôle primordial et indispensable à former la personnalité de l'apprenant, à enrichir ses connaissances, à satisfaire ses goûts, ses besoins et ses intérêts et enfin à modifier ses comportements. De plus, elle l'aide à acquérir de diverses expériences dans les différents domaines de la vie quotidienne (Gabr, 2007: 22).

Dans ce sens, la compréhension écrite permet aux apprenants de conserver les informations facilement. Ainsi, elle leur permet de les activer et réviser. Elle leur permet d'effectuer différentes activités en classe. En fin, la compréhension écrite permet d'enrichir le vocabulaire et la grammaire de la langue chez les apprenants pendant les pratiques variées de la langue écrite (Mohamed, 2005: 108).

\subsection{2. Étapes de la compréhension écrite}

Selon Cuq et Gruca (2005: 167) cité par Badary (2017: 251260 , il ya trois étapes de la compréhension écrite:

- La prélecture: la prélecture ou la préparation à la lecture est une phase essentielle pour favoriser l'accès au texte et faire des hypothèses. C'est le temps de la mise en situation où le lecteur s'informe de l'intention du sujet, de la structure ou du genre du texte.

- La lecture: C'est la phase ou le lecteur lit le texte et travaille sur les différentes stratégies qui lui accordent de gérer son 
compréhension en faveur de son intention la lecture. Ainsi, il peut vérifier les hypothèses dégagées lors de la phase de prélecture, organiser les informations qui se produisent, traiter ces informations en les comparant à ses connaissances antérieures et en venir à se poser des questions nouvelles qui conduiront a d'autres hypothèses. Le lecteur fait constamment un retour sur sa démarche de la lecture dans le but d'établir la signification d'un texte.

- La post lecture: C'est la phase ou le lecteur objective sa démarche de compréhension. Il pense sur ce qui a été fait, comment cela a été fait, sur 1 les difficultés rencontrées et sur ses acquisitions. Les apprenants vont alors s'en appuyer pour accomplir une tache ou atteindre des buts assujettis.

\subsubsection{Le lien entre la compétence de la compréhension écrite et les compétences langagières}

La compétence de la compréhension écrite s'apprécie dans des situations de communication et la priorité dévolue aux habilites. Savoir lire, écouter, parler et écrire s'exerce sur un corpus plus quotidien.

\section{- Le lien entre la compréhension écrite et l'expression orale}

Les recherches attestent qu'il existe un rapport rigide entre communication verbale et la compréhension écrite. Ce rapport est paru dans plusieurs domaines :

- Les types de l'expression orale sont un fondement pour l'enseignement de la compréhension écrite.

- Les faibles habitudes de l'expression orale doivent être modifiées avant le déroulement de l'enseignement de la lecture.

- Le lexique du texte exploré doit être appris oralement avant l'enseignement de la lecture.

\section{- Le lien entre la compréhension écrite et la production écrite et}

D'une manière globale, le bon lecteur est un bon rédacteur et réciproquement. $\mathrm{La}$ lecture alimente le rédacteur par des idées récentes. La bonne lecture favorise la bonne production écrite. Il est donc nécessaire de faire acquérir aux apprenants des stratégies de la lecture pour passer progressivement à la production écrite, mais, certains études indiquent que la pratique d'activité d'écrits favorise a l'apprenant de progresser en lecture et d'obtenir de bonnes notes aux épreuves d'assimilation de l'écrit. Ainsi, l'enseignement de l'écrit passe par 
la lecture. La lecture est l'écriture organisent les faces d'un même exercice.

\subsubsection{Compétences et niveaux de la compréhension écrite}

Sans doute, la compréhension écrite est la condition de toute éducation, parce que l'étudiant ne peut rien apprendre s'il ne sait pas comprendre ce qu'il est. Cette compréhension requiert une vaste gamme de compétences :

Chérif (2002: 103-109) cité par Abdel Aal (2016: 39-42) classifie les compétences de la compréhension écrite comme le suivant:

- Créer un titre ou une idée générale du texte écrit.

- Déterminer les idées pour chaque paragraphe du texte.

- Diviser le texte écrit en paragraphes en les reliant.

- Résumer le texte en une phrase correcte en un temps limité.

- Distinguer les idées principales et les idées secondaires.

- Comprendre le thème du texte lu.

- Saisir le sens implicite.

- Pouvoir déterminer le genre du texte.

Les compétences de la compréhension écrite selon Mahfouz (2009: 81-86) sont:

- Suivre l'organisation du texte.
- Comprendre les idées principales.

- Reconnaître la phrase.

- Reconnaître le paragraphe.

- Choisir le titre.

- Résumer l'idée principale.

- Reconnaître les relations entre les phrases.

- Reconnaître les idées et leur organisation.

- Choisir le titre convenable du texte.

- Résumer ce qui a été lu.

Pour Sobhi (2010 : 63-65),

les compétences de la

compréhension écrite sont :

- Examiner la cohésion et la cohérence des phrases

- Identifier l'idée principale et les idées secondaires du texte

- Saisir le sens d'une phrase à travers le contexte

- Saisir profondément le sens implicite du texte

- Dégager les mots clés et leur donner une signification adéquate

- Comparer les nouvelles informations aux connaissances antérieures

- Donner un titre convenable au texte.

- Diviser le texte en paragraphes et connaître les liens entre leurs idées. 
En ce qui concerne les niveaux de la compréhension écrite:

D'après la classification de Smith et Barrett (1974:52-58) cité par Abdel Aal (2016: 43-44), Badary (2017: 80-81), Elsafy ( 2005: 56-73), Sobhi (2010: 66-68), on peut indiquer les niveaux de la compréhension écrite comme le suvant:

\section{- La compréhension littérale du} texte: il s'agit pour le lecteur de prélever dans le texte les indicesexplicites qui permettront la construction du sens. C'est une activité de tri quitend à isoler et mémoriser parmi tous les indices explicites du texte ceux quisont utiles à cette construction. Cette compréhension est la plus réussie par lesapprenants comme le prouvent les évaluations nationales.

\section{- La compréhension} inférentielle: cette compréhension se divise en logique et pragmatique. Pour la compréhension logique, le lecteur relève des informations éparses dans le texte, les met en relation afin de déduire de nouveaux éléments de sens. Pour la compréhension pragmatique, Le lecteur met en relation des informations contenues dans le texte avec ses propres connaissances, sa culture personnelle afin de faire du sens.

- La compréhension fine: le lecteur est alors capable de " lire entre les lignes "), c'est à dire de percevoir l'implicite du texte.

- La compréhension critique: C'est porter un jugement sur le texte lu. Autrement dit, La compréhension critique doit comprendre la critique de tout le texte du point de vue linguistique et sémantique pour arriver à l'évaluer. Le lecteur ou la lectrice évalue l'exactitude du texte lu à la lumière de ses connaissances. Pour développer les compétences de la lecture critique, on doit saisir le style de l'auteur.

- La compréhension créative: C'est le niveau de compréhension qui permet au lecteur ou à la lectrice d'appliquer les différentes significations trouvées dans une lecture, à sa vie personnelle, de créer de nouvelles idées, de proposer de nouvelles séquences intellectuelles. Les compétences de la compréhension créative nécessitent des additions au texte.

En somme, cette classification fondée sur les processus cognitifs a joué un rôle 
important en éducation car elle a permis d'élargir l'éventail des questions possibles à poser aux apprenants après la lecture d'un texte.

\subsection{La production écrite}

On expose dans les lignes suivantes la notion de la production, son importance, ses compétences, ... etc.

\subsubsection{Notion de la production écrite}

La production écrite se définit comme une tache complexe qui peut être envisagée sous différents aspects (Zesiger, 1995: 54) cité par Nassar (2017: 16):

- Communicationnelle: le fait de s'inscrire dans une démarche de communiquer en choississant de recourir a l'écrit;

- Sémantique: le sens du message de l'écrit;

- Linguistique: les mots, les termes utilisés, leur organisation;

- Socio-culturel: les règles et les normes de communication institutionnellement inscrites.

- pragmatique: les connaissances sur les intentions de communication.

De plus, l'écriture considère un acte signifiant qui amène l'apprenant à exprimer ses idées, ses sentiments, ses besoins, ses intérêts pour les communiquer avec les autres. Cette forme de communication exige la mise en oeuvres des habiletés et des stratégies que l'apprenant sera appelé à maîtriser graduellement au cours de ses apprentissages.

\subsubsection{Importance de la production écrite}

Ecrire pour communiquer des informations; l'écrit est un objet vivant servant à communiquer aux sens larges, c'est-à-dire, informer, mémoriser, prescrire, provoquer et exprimer des actions, des attitudes ou des sentiments, il est une nécessité sociale pour transmettre les pensées et connaître, la pensée d'autrui. L'écriture sert à des fins d'expression, de création, de communication et d'apprentissage tout à la fois. Apprendre à rédiger des textes variés permet de répondre à des besoins personnels, éducatifs et sociaux (Al Banoui, 2018: 17).

Ecrire est un acte de communication qui présuppose qu'il existe un destinataire. L'acte d'écrire remplit, dans la vie quotidienne des fonctions liées à celles de la lecture: écrire, c'est produire du texte à lire, c'est émettre un message qui sera reçu au travers d'un acte de lecture et ce n'est qu'à travers cet acte de lecture que l'acte d'écriture peut prendre sens. Réciproquement, la personne qui apprend à lire ne pourra donner du sens à son acte de lecture qu'à 
travers le sens qu'elle donne à ses propres activités d'écriture.

Généralement, l'étude de Wafy (2014: 31), déduit l'importance de l'expression écrite qui réside dans l'acte de (d'):

- Constituer une immense mémoire du monde, des peuples, des nations et des individus.

- Permettre de construire le savoir et de le transmettre.

- Être un puissant moyen d'expression et de communication.

- Être au centre de l'activité scolaire de l'apprentissage.

- Être dans la communication, de plus en plus complémentaire avec l'image (publicité, bande dessinée, revue, et livre scolaire).

\subsubsection{Compétences de la production écrite}

D'après les études d'Al Banoui (2018), Abd El Salam (2007), Ali (2014), Wafy (2014), on peut aborder ces compétences comme suit:

- Pré écrire : cette étape comporte les compétences suivantes:

- Organiser les idées.

- Activer les connaissances antérieures.

- Activer le vocabulaire.
- Utiliser les mots donnés.

- Pendant l'écriture: cette étape comporte les compétences suivantes:

- Ecrire un premier paragraphe.

- Cohérence des idées.

- Éviter les fautes grammaticales et lexicales.

- Après l'écriture (la révision): cette étape contient les compétences suivantes:

- Vérifier l'orthographe grammaticale des mots en tenant compte des indices fournis par le texte.

- La vérification de la cohérence et de la structure du texte.

\subsection{4. Évaluation de la} production écrite

L'évaluation du savoir-écrire devrait s'effectuer en utilisant une tâche la plus réelle possible qui nécessite que l'apprenant effectue la planification, la rédaction et la révision de son texte. Cette tâche doit présenter une intention de communication à destinataire potentiel. Toutes évaluations d'une production écrite devraient tenir compte des connaissances déclaratives, procédurales et conditionnelles nécessaires à sa réalisation.

En somme, en production écrite, l'évaluation sera 
principalement d'ordre formative, diagnostique et sommative. Il faut tout d'abord décider ce qui doit-être évalué et pourquoi on l'a évalué. Les résultats d'apprentissage sont le point de départ vers cette décision. Ils orienteront le choix des composantes de l'écriture qui seront évaluées.

Selon Al Banoui (2018: 2425), il y a plusieurs aspects d'évaluation de la production écrite:

- Le contenu: c'est le des idées ou des informations à transmettre.

- Le style: c'est l'emploi de differentes structures linguistiques afin de donner au texte une touche originale et personnelle pour maintenir l'intérêt du lecteur.

- Les règles de langue: c'est la capacité l'utilisateur/l'apprenant d'utiliser les structures grammaticales et lexicales.

\section{Résultats de la recherche}

L'hypothèse de cette recherche réside qu'Il y a une différence significative statistiquement au niveau $\mathrm{P}(\leq$ $0,05)$ entre les moyennes des notes des étudiants de l'échantillon de la recherche aux pré/post tests des compétences de la communication écrite (compréhension écrite et production écrite) en faveur du post test.

Pour vérifier cette hypothèse, le chercheur a utilisé Test «t» pour démontrer la signification statistique des différences entre les moyennes des notes des étudiants $\mathrm{du}$ groupe expérimental de la recherche. Il a également calculé la moyenne, l'écart type, le degré de la liberté, et la valeur de « T » au test des compétences de la communication écrite (compréhension écrite et production écrite) avant et après l'application du programme d'entrainement. Le tableau N. ${ }^{\circ}$ (2) indique ces résultats. 
Tableau $\mathbf{N}^{\circ}$ (2) «Test «t» de la signification des différences entre les moyennes de notes d'étudiants du groupe expérimental de la recherche au pré/post-test des compétences de la communication écrite (compréhension et production)

\begin{tabular}{|c|c|c|c|c|c|c|c|}
\hline 递 & Application & N. ${ }^{\circ}$ & M & E.T. & D.L. & 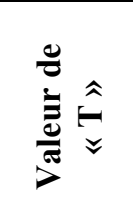 & 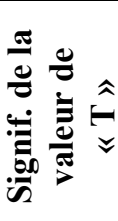 \\
\hline 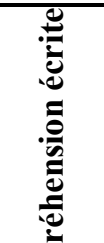 & Pré & 30 & 3.2667 & 1.5071 & \multirow[t]{2}{*}{29} & \multirow[t]{2}{*}{41.1593} & \multirow[t]{2}{*}{$\begin{array}{l}0.05 \\
\text { N.S. }\end{array}$} \\
\hline $\begin{array}{l}\tilde{U} \\
\dot{1} \\
\end{array}$ & Post & 30 & 14.9667 & 2.1573 & & & \\
\hline \multirow{2}{*}{ 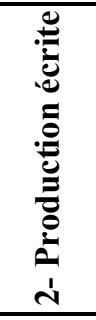 } & Pré & 30 & 3.3667 & 1.2994 & & \multirow{2}{*}{38.9667} & \multirow{2}{*}{$\begin{array}{l}0.05 \\
\text { N.S. }\end{array}$} \\
\hline & Post & 30 & 15.4000 & 2.1592 & 29 & & \\
\hline \multirow{2}{*}{ Total } & Pré & 30 & 6.6333 & 2.3995 & \multirow{2}{*}{29} & \multirow{2}{*}{49.6484} & 0.05 \\
\hline & Post & 30 & 30.1667 & 3.3742 & & & N.S. \\
\hline
\end{tabular}

\section{Légende}

$\mathrm{N}^{\circ}$ : Nombre d'étudiants

M.: Moyenne des Notes

D.L.: Degré de liberté

E.T.: Écart type

T. : Valeur de « $T »$

S.: Signification

Le tableau ci-dessus démontre qu'il existe une différence statistiquement significative au niveau $P \leq 0.05$ entre les moyennes des notes d'étudiants de l'échantillon de la recherche au pré/post test des compétences de la communication écrite (compréhension et production) en faveur du post test. Cette valeur met l'accent sur la progression dûe à l'utilisation du programme d'entrainement et son impact observable sur le développement des compétences de la communication écrite. Donc, l'hypothése de la recherche actuelle a été vérifiée et achevée. 
Tableau $\mathbf{N}^{\circ}$ (3):Taille de l'effet du programme d'entrainement sur le développement compétences de la communication écrite (compréhension écrite et production écrite)

\begin{tabular}{|c|c|c|c|c|c|c|}
\hline 苞 & Application & N. ${ }^{0}$ & $\begin{array}{c}\text { Valeur } \\
\text { de } « T »\end{array}$ & $\eta 2$ & D.L. & T.E \\
\hline 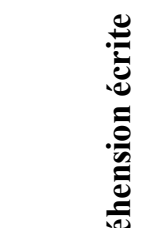 & Pré & 30 & \multirow[t]{2}{*}{41.1593} & \multirow[t]{2}{*}{0.9832} & \multirow[t]{2}{*}{29} & \multirow[t]{2}{*}{ élevé } \\
\hline $\begin{array}{l}\bar{\Xi} \\
\dot{U} \\
\dot{u}\end{array}$ & Post & 30 & & & & \\
\hline \multirow{2}{*}{ 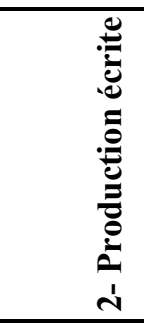 } & Pré & 30 & \multirow[t]{2}{*}{38.9667} & \multirow[t]{2}{*}{0.9832} & & \multirow{2}{*}{ élevé } \\
\hline & Post & 30 & & & 29 & \\
\hline \multirow{2}{*}{ Total } & Pré & 30 & \multirow[t]{2}{*}{49.6484} & \multirow[t]{2}{*}{0.9884} & \multirow{2}{*}{29} & \multirow{2}{*}{ élevé } \\
\hline & Post & 30 & & & & \\
\hline
\end{tabular}

\section{Légende:}

T. : Valeur de « $\mathrm{T} »$

ๆ2: Carré d'Eta.

T.E.: Taille de l'effet

Le tableau ci-dessus démontre cette taille de l'effet qui était (0.98), donc, elle est significative et élévée. On trouve que (98\%) des changements qui se passent à la variable dépendante des compétences de la communication écrite (compréhension et production) reviennent à la variable indépendante (Le programme d'entrainement). Alors, ce programme a un grand effet, donc, il efficace, parce que on peut mesurer l'impact d'un programme d'après son effet.

\section{Discussion des résultats}

À la lumière des résultats de la présente recherche, on trouve qu'il existe un développement observable dans la performance des étudiants de l'échantillon, les étudiants de la deuxième année au 
cycle secondaire concernant les compétences de la communication écrite (compréhension écrite et production écrite) aux pré/post tests en faveur du post test. Cela démontre l'effet de l'emploi du programme d'entrainement.

\section{Ce résultat peut être interprété comme suit:}

- Le programme d'entrainement a créé une atmosphère qui favorise l'acquisition des compétences de la communication écrite (compréhension écrite et production écrite).

- Le programme d'entrainement a aidé les apprenants à utiliser la langue française dans la vie courante.

- Les interactions sociales faites entre les apprenants au sein des groupes leur ont donné l'occasion de dialoguer, de collaborer, d'exploiter un savoir- faire et de varier des acquis communicatifs (ici l'utilisation de la langue française dans la vie quotidienne).

\section{Recommandations de l'étude}

Selon les résultats de la recherche actuelle, on peut présenter les recommandations suivantes :

- Il faut recourir aux méthodes d'enseignement et aux programmes d'entrainement qui encouragent l'interaction soiale entre les apprenants et leurs enseignants.

- Il est nécessaire d'elaborer des programmes d'entrainement afin d'aider l'utilisateur/apprenants à utiliser la langue française dans les différentes/nouvelles situations de communication.

- Il faut prendre en considération les recommandations du Cadre Européen Commun de Références pour les Langues qui a mis en valeur la fonction du langage dans le contexte social dont le but est de repenser les objectifs et les méthodes d'enseignement des langues pour fournir une base commune pour la conception des programmes d'entrainement, de diplômes et de certificats.

\section{Suggestions de la recherche}

Cette recherche propose d'autres recherches telles que:

- Elaborer des recherches en utilisant un programme d'entrainement basé sur l'approche actionnelle du CECRL pour développer les compétences communicatives chez les étudiants du cycle secondaire.

- Effet d'un programme d'entrainement sur le développement quelques compétences de la communication orale et la motivation chez les étudiants de la section de français aux 
facultés de pédagogie.

- Effectuer des recherches utilisant un programme basé sur la théorie pragmatique pour développer quelques compétences de la lecture critique chez les étudiants de la section de français aux facultés de pédagogie.

- Elaborer des recherches utilisant un programme basé sur l'approche fonctionnelle sur le développement quelques compétences auditives.

\section{Conclusion}

Tout d'abord, la présente recherche s'intersse à développer les compétences de la compréhension écrite et la production écrite. Cette recherche est incitée par le niveau faible des étudiants de la $2^{\text {ème }}$ année secondaire en ce qui concerne ces compétences. Enfin, l'objectif primordial de cette recherche est de mettre en évidence l'efficacité du programme d'entrainement sur le développement de ces compétences de la communication écrite (la compréhension écrite et la production écrite) chez les étudiants du cycle secondaire.

Dans ce contexte, ce programme d'entrainement aide l'apprenant à metttre l'accent sur toutes ses compétences pour accomplir plusieurs activités. En revanche, ce programme comporte des activités d'entrainement, l'apprenant s'entraine à les faire afin d'être apte à employer la langue dans les nouvelles situations communicationnelles.

Finalement, après avoir analysé quantitativement les résultats obtenus, il semble que le programme d'entrainement était efficace. Dès lors, les résultats de la présente recherche sont encourageants en ce qui a trait au développement des compétences de la communication écrite (compréhension et la production) chez les étudiants de la deuxième année secondaire.

\section{Bibliographie}

Abdel-Aal, M. (2016). Efficacité de la stratégie de l'enseignement réciproque pour le développement des compétences de la compréhension des textes lus et l'attitude vers la langue française chez les étudiants du cycle secondaire d'Al-Azhar, Thèse de Magistère, Faculté de pédagogie, Université de Mansourah.

Abdel Salam, M. (2007). L'effet de l'utilisation du Brainstorming sur le développement de quelques compétences de l'expression écrite chez les étudiants de la première année à la faculté de Pédagogie de Minia section de Français. Thèse de Magistère. Faculté de Pédagogie. Université de Minia. 
Abensour, C. (1998). Les efficaces, pratique de la communication écrite. Paris: Nathan.

Al Banoui, M. (2018). Efficacité d'une unité pour enrichir le vocabulaire basée sur l'apprentissage génératif en vue de développer les compétences de l'expression écrite chez les étudiants de section de français. Thèse de Magistère. Faculté de pédagogie université d'Al Azhar.

Ali, I. (2014). Impact d'un dispositif hypermédiatique sur le développement de quelques compétences langagières chez les étudiants de section de Français à la faculté de Pédagogie de Sohage. Thèse de Doctorat. Faculté de Pédagogie. Université de sohag.

Alrabdi, E. (2011). Quelle méthode faut-il adopter pour l'enseignement/apprentissage de l'oral? Université du Yarmouk. Département de langues modernes. Tiré de revistas.ucm.es/index.php/DI

DA/article/download./36308/ 35153.

Awad, H. (2013). Utilisation de la grammaire récit pour développer les compétences de l'écriture chez les élèves du cycle préparatoire aux écoles francophones. Thèse de Magistère. Faculté de pédagogie. Université d'Ain Chams.
Badary, E. (2017). Efficacité d'un programme basé sur l'approche interculturelle dans le développement de quelques compétences de la compréhension écrite chez les futurs enseignants de FLE. Thèse de Magistère. Faculté de pédagogie. Université de Sohag.

Chalabi, Ch. (2014). Efficacité de la pédagogie de maîtrise pour corriger certaines erreurs communnes en expression écrite chez les étudiants de la section de Français, à la faculté de pédagogie. Thèse de Magistère. Faculté de pédagogie d'Al-Arich. Université du Canal de Suez.

Conseil de la coopération culturelle (1998). Apprentissage et usage des langues dans le cadre européen. Strasbourg: Edition du conseil de l'Europe. Tiré de https://books.google.com.eg.

Conseil de l'Europe (2001). Cadre européen commun de référence pour les langues: Apprendre, Enseigner, Évaluer. Paris: Didier.

Cuq, J. et Gruca, I. (2005). Cours de didactique de français langue étrangère et seconde. Grenoble: PUG.

Deschênes, A. (1988). La compréhension et la production de textes. Québec: Presses de l'université du Québec. 
Delmson, F. (2005). Enseigner le FLE. Pratiques de class. Paris: Belin.

El Ahwal, W. (1991). Développement de certaines capacités d'expression écrite en langue française chez les étudiants des facultés de pédagogie, Thèse de Doctorat. Faculté de pédagogie de Damiette. Université de Mansourah.

El-Chahat, G. (2000). Efficacité du programme proposé pour le développement des compétences de lecture d'étude chez les étudiants des départements de français des facultés de pédagogie. Thèse de Doctorat, Faculté de pédagogie. Université de Mansourah.

El Safy, M. (2005). L'emploi de l'enseignement réciproque en vue de développer les compétences de la compréhension en lecture nécessaires aux étudiants de la faculté de pédagogie (Étude expérimentale). Thèse de Magistère, Faculté de pédagogie. Université de Ménoufya.

Esmat, N. (2016). L'efficacité d'un programme basé sur l'apprentissage Hypride pour développer la production écrite et les compétences métacognitives chez les étudiants de la section de français, faculté de Pédagogie à Assiout. Thèse de Doctorat. Faculté de Pédagogie. Université d'Assiout.

Fouad, R. (2014). Efficacité de l'emploi de la stratégie des cartes sémantiques sur le développement des compétences de la compréhension lecturale chez les étudiants de faculté de pédagogie-section de français. Thèse de Magistère, Faculté de pédagogie, Université de Mansourah.

Gabr, M. (2007). L'effet d'un programme assisté par ordinateur pour développer les compétences de la compréhension écrite en français chez les élèves de première année secondaire. Thèse de Magistère, Faculté de pédagogie, Université de Minia.

Gauthier,

C.

L'enseignement explicite. Fondements et pratiques. CRIFPE, Université Laval.

Giasson, J. (2007). La compréhension en lecture, 3ème édition. Boecket Larcier s. a, Paris.

Mahfouz, H. (2009). L'efficacité d'un programme proposé des activités pré-scolaires pour développer les compétences de compréhension de la lecture silencieuse en français au cycle primaire. Thèse de Magistère. Institut d'études 
pédagogiques. Université du Caire.

Mohamed, S. (2005). Élaboration d'un modèle d'apprentissage fondé sur l'autonomie pour dèvelopper la compétence de la communication écrite en FLE comme deuxième langue étrangère du chez les élèves du cycle secondaire. Thèse de Doctorat. Faculté de Pédagogie. Université de Tanta.

Nassar, A. (2017). Efficacité de l'utilisation de l'apprentissage par projet sur le développement des compétences de l'expression écrite chez les étudiants de la section de français. Thèse de Magistère. Faculté de Pédagogie. Université d'Al Azhar.

Noyon, Ch. (2011). Les interactions écrites en ligne en classe de français langue étrangère: élaboration d'un dispositif, analyse des discours produits et analyse des représentations. Thèse de
Doctorat. Université Catholique de Louvain: Faculté de Philosophie, Arts et lettres.

Sobhi, W. (2010). Effet de la stratégie du brainstorming sur le développement des compétences de la lecture critique chez les étudiants du département de français aux facultés de pédagogie. Thèse de Magistère, Faculté de pédagogie. Université de Ménoufya.

Wafy, M. (2014). Efficacité d'un programme proposé pour l'enseignement de la grammaire basé sur l'approche fonctionnelle sur le développement des compétences de l'expression écrite en français chez les étudiants de la deuxième année secondaire d'Al Azhar. Thèse de Magistère. Faculté de Pédagogie. Université d'Al Azhar.

Weiss, F. (2002). Jouer, communiquer, apprendre. Paris: Hachette. 